Recepción: 05/11/2018

Aceptación: 10/01/2019

Publicación: 05/02/2019

Ciencias económicas y empresariales Artículo de revisión

\title{
Ámbitos de investigación de la carrera de administración de empresas: tecnologías avanzadas de gestión empresarial y modelado
}

\section{Fields of research in the career of business administration: advanced business management and modeling technologies}

\section{Áreas de pesquisa na carreira de administração de empresas: gerenciamento avançado de negócios e tecnologias de modelagem}

\author{
Jorge Oswaldo Quevedo-Vázquez ${ }^{\mathrm{I}}$ \\ joquevedov@ucacue.edu.ec \\ Mercedes Lucia Neira-Neira ${ }^{\text {II }}$ \\ mlneiran@ucacue.edu.ec \\ Tania Maricela Villarreal-Cherrez III \\ tmvillarrealc@ucacue.edu.ec
}

\section{Correspondencia: joquevedov@ucacue.edu.ec}

${ }^{\text {I }}$ Magíster en Administración de Negocios, Diploma Superior en Práctica Docente Universitaria, Economista, Licenciado e Economía y Finanzas, Subdirección de Posgrados Universidad Católica de Cuenca, Cuenca, Ecuador.

II Doctora en Jurisprudencia, Diploma Superior en Pedagogías Innovadoras, Especialista en Diagnostico Intelectual, Magister en Desarrollo de la Inteligencia y Educación, Abogado de los Tribunales de Justicia de la Republica, Licenciada en Ciencias Jurídicas y Sociales, Profesora de Educación Media en la Especialidad de Pedagogía, Profesor de Educación Primaria-Nivel Técnico Superior, Docente Universidad Católica de Cuenca, Cuenca, Ecuador.

III Magíster en Auditoria de Gestión de la Calidad, Diploma Superior en Auditoria de Gestión de la Calidad, Especialista en Auditoria de Gestión de la Calidad, Ingeniero Empresarial, Auditor, Contador Público, Docente Universidad Católica de Cuenca, Cuenca, Ecuador. 


\title{
Resumen
}

Actualmente la Tecnología es el motor más importante para la prosperidad de las empresas, para ello estas deben definir el desarrollo de nuevos productos hacia una cultura de innovación. En este trabajo se describen las Tecnologías avanzadas de Gestión Empresarial y modelado. Para cumplir con este propósito, se propuso una metodología que combina elementos de la investigación documental y descriptiva de carácter retrospectivo. El corpus teórico, se estructuro a partir de los planteamientos evaluados y examinados por estudiosos del tema, así como la experiencia de investigadores del área de la tecnología e información desde la web. El análisis e interpretación de las teorías permitieron presentar entre sus conclusiones que la integración de la tecnología en las labores empresariales hará que se den mejores resultados y se genere una mejor aceptación en los diferentes mercados, así como generar la optimización de los recursos y, por tanto, mayor beneficio para la empresa.

Palabras clave: Tecnología; gestión empresarial; modelado y empresarios.

\begin{abstract}
Currently the technology is the most important engine for prosperity of enterprises, so these must define the development of new products to a culture of innovation. This work describes the advanced technologies of modeling and business management. For this purpose, proposed a methodology that combines elements of the retrospective documentary and descriptive research. The theoretical corpus, I will structure from the approaches tested and examined by scholars, as well as the experience of researchers in the field of technology and information from the web. The analysis and interpretation of the theories allowed present among its conclusions that the integration of technology in the business work will make best results den and a better acceptance is generated in different markets, as well as to generate the optimization of resources and, therefore, greater benefit for the company.
\end{abstract}

Key words: Technology; business management; modeling; and entrepreneurs. 


\section{Resumo}

Atualmente a tecnologia é o motor mais importante para a prosperidade das empresas, para isso devem definir o desenvolvimento de novos produtos para uma cultura de inovação. Este artigo descreve as tecnologias avançadas de Business Management e modelagem. Para cumprir este propósito, foi proposta uma metodologia que combina elementos de pesquisa documental e descritiva de caráter retrospectivo. O corpus teórico, é estruturado a partir das abordagens avaliadas e examinadas pelos estudiosos do assunto, bem como a experiência de pesquisadores na área de tecnologia e informação da web. A análise e interpretação das teorias possibilitaram apresentar, entre suas conclusões, que a integração da tecnologia nas tarefas de negócios levará a melhores resultados e melhor aceitação nos diferentes mercados, além de gerar a otimização de recursos e, Portanto, maior benefício para a empresa.

Palavras-chave: Tecnologia; gestão empresarial; modelagem e empreendedores.

\section{Introducción}

Días, Gonzales y Ruiz (2005) exponen que desde los años 80, la tecnología ha jugado un papel fundamental en los cambios de los paradigmas de cómo se hacen los negocios. Todas las organizaciones se encuentran sumergidas en un escenario cambiante, en la cual la organización que no se alinee con la tecnología irá perdiendo clientes y lentamente, saldrán del mercado. Han obligado en muchas ocasiones a la adopción de tecnologías, para sobrevivir y continuar operando de forma eficiente en un mercado global y competitivo; porque la competencia estará un escalón adelante, y finalmente sólo tendrá una ventaja. Unos de los primeros pasos para cumplir los principales factores claves para el éxito, es el uso eficiente de las Tecnologías de Información.

Ravina, Villena y Gutiérrez (2017), mencionan que: actualmente, se vive en una economía globalizada e interconectada, donde se establece como el verdadero motor de la innovación a la transferencia de conocimiento científico, siempre y cuando haya un tejido industrial en las tecnologías de la información y la comunicación (TICS), que da a conocer cómo estas tecnologías se han convertido en un elemento indispensable para responder de forma ágil a los cambios del entorno. Es importante abordar el estudio concreto de este tipo de empresas puesto que presenta cierta determinación. 
Núñez (2011) manifiesta que, en las sociedades modernas, la ciencia y la tecnología son factores de gran peso socioeconómico. En los mercados internacionales los productos con un fuerte componente tecnológico son altamente demandados, por lo que las empresas que buscan desarrollar la competitividad requieren incorporar tecnología moderna e impulsar la innovación. Las empresas se dirigen a optimizar sus modelos de gestión y a adquirir o desarrollar tecnología. La definición de estrategias es fundamental para lograr el objetivo organizacional, debe orientarse a incorporar políticas organizacionales. La gestión tecnológica es una herramienta para concretar la misión de una empresa innovadora mediante sus fuerzas internas de creatividad o el uso de transferencias tecnológicas.

En palabras de autores como Barragán y Vela (2015), el desarrollo de las tecnologías de información en los negocios ha llamado la atención ya que son tan importantes en las empresas e industrias porque aportan éxito y nivel de competitividad alto. Su utilización puede hacerle la vida más fácil a las personas y a los negocios. La tecnología moderna puede ayudar a que el negocio trabaje eficientemente, reducir tiempos en los procesos internos y aumentar la comunicación entre empleados, clientes o proveedores.

Zapata, Arango y Adarme (2010), aseguran que el cambio tecnológico producido por la revolución de la tecnología marca la diferencia entre una civilización desarrollada y otra en vías de desarrollo, por lo tanto, una organización que integra tecnología en sus actividades es una empresa subdesarrollada en el mundo actual. La aplicación de las (TICS) permite simplificar las funciones rutinarias y dedicar tiempo a las funciones de la organización, se presenta como elementos esenciales para mejorar la competitividad de la organización.

Desde la perspectiva de Díaz (2006), el conocimiento debe ser concebido de manera adecuada, oportuna, construida, sistematizada, compartida con labores que constituyen retos de la gerencia como los tradicionales intereses de disponer de la mejor tecnología, políticas y recursos humanos para maximizar la producción y las utilidades. Para tener éxito se requiere un nuevo marco conceptual, que se refiera al conocimiento en las organizaciones y las Tecnologías de Información y Comunicaciones (TIC) como parte de una estrategia más amplia en dimensiones sociales y culturales. 
Se buscan recomendaciones para aquellas empresas que asumen retos de permanencia en el mercado, como extraer el conocimiento actual y potencial requerido en sus negocios, rescatar los procesos empresariales como una fuente de ventaja competitiva, apoyando el cumplimiento de estos objetivos con las Tecnologías de Información y Comunicación.

En pensamiento de Ramírez y Vega (2015), las organizaciones deben establecerse en un mercado globalizado y competitivo. El uso efectivo de la plataforma tecnológica, permitirá agilizar los procesos administrativos, interactuar con otras organizaciones obteniendo un valor agregado en sus productos o servicios. Castel y Ramírez (2017), mencionan que la aparición de nuevas formas organizativas tales como las empresas en red y la aparición de la tecnología de la información y la comunicación (TIC) son algunas de las transformaciones producidas en el entorno empresarial de los últimos años. Especialmente para las pequeñas y medianas empresas (PYMES), la competencia en los mercados ha obligado en muchas ocasiones a la adopción de estas tecnologías. De esa manera ocupan un lugar elevado las dedicadas al caso de las (PYMES), puesto que estas organizaciones generan una parte importante del Producto Interior Bruto, son generadoras de empleo, agentes de estabilización social y fuentes de innovación, especialmente en los países en desarrollo.

Las ideas dadas por los autores Perozo y Nava (2005), muestran que las empresas han decidido invertir en mejores recursos y optimizar los ya implementados, mediante la administración adecuada de factores internos tratando de lograr un elevado desempeño en el mercado. Ante los desafíos de organizar la producción al aplicar conocimientos a entornos cada vez más industrializados, la gestión tecnológica surge como una de las capacidades con las que puede contar la organización actual, para administrar la tecnología que se implementa dentro de las diferentes áreas organizativas, recursos humanos, finanzas, investigación, desarrollo, procedimientos informativos y gestión gerencial. Con el reconocimiento de la necesidad de adquirir elementos diferenciadores y gracias al desarrollo social, económico y político, las empresas optan por asumir nuevos riesgos y tener la mejor decisión en ambientes que influyen en avances tecnológicos y en viabilidad de aplicación de diferentes procesos. Para esto, la gestión de la tecnología integra elementos innovadores con el fin de generar ventajas competitivas a lo largo de la organización con su entorno, convirtiéndose en el mayor impacto producido por las TICS 
con el contexto empresarial espontaneó.

Por lo antes manifestado, autores como Barragán y Vela (2015), en su discurso expresan que el desarrollo de las tecnologías de información en los negocios ha llamado la atención ya que son tan importantes en las empresas e industrias porque aportan éxito y nivel de competitividad alto. Su utilización puede hacerle la vida más fácil a las personas y a los negocios. La tecnología moderna puede ayudar a que el negocio trabaje eficientemente, reducir tiempos en los procesos internos, aumentar la comunicación entre empleados, clientes o proveedores. Ante esta posición, en este artículo se caracteriza en el ámbito de investigación de la carrera de administración de empresas las tecnologías avanzadas de gestión empresarial y modelado

\section{Desarrollo}

El profesor Perel (2015) expresa que la tecnología ha desafiado no solo al tiempo y a la distancia sino también los fundamentos científicos sobre la mente y la inteligencia. Si bien como se ha señalado, la evolución tecnológica abarca a todas las áreas del saber humano, es frecuentemente compartido entre las nuevas tecnologías de propósito general que se destacan en la actualidad se encuentran las biotecnologías, las de nuevos materiales, las energéticas, la robótica y las tecnologías de la información y comunicación (TICS). Para ello, plantea que se debe tomar en cuenta los nuevos materiales tecnológicos, tales como:

- Plataformas digitales móviles.

- Crecimiento del Software en línea como un servicio.

- Crecimiento de la computación en la nube.

El buen administrador debe buscar la información donde se encuentra y no sólo manejarse con la información que más fácilmente se consigue. Debe ser capaz de medir aún aquello que es difícil de medir (satisfacción de empleados, clima laboral, satisfacción de consumidores y clientes).

Esa información debe enfocar, ante todo, los factores críticos del éxito organizacional, teniendo siempre en cuenta la misión, visión y valores de la organización. (Medellín Cabrera, 2010)

\section{2}

Pol. Con. (Edición núm. 30) Vol. 4, No 2, febrero 2019, pp. 407-424, ISSN: 2550 - 682X 
Sin embargo, más allá de todo el avance tecnológico que pueda existir no se debe olvidar que por sobre todo está el ser humano con sus sentimientos, defectos y virtudes y que un abrazo nunca va a poder ser reemplazado por ninguna máquina. (Ramírez y Vega, 2015).

Al respecto, Marcovitch (2001) desarrolla el tema de la modernización tecnológica conectada al proceso de desarrollo de una sociedad. Esta deberá estar vinculada al uso de tecnologías adecuadas a sus materias primas, sus recursos humanos, sus necesidades y su realidad, para encontrar soluciones innovadoras.

EL autor Zamora, (2013), manifiesta que la adopción de estas nuevas tecnologías no debe verse únicamente como una amenaza en la destrucción de la cadena de valor tradicional, sino como una potente herramienta de la competitividad de las empresas. Por su parte Martínez (2006), menciona que no existen países desarrollados ni países subdesarrollados, sino simplemente países que saben administrar la tecnología existente y sus recursos actuales potenciales, y países que todavía no lo saben lo cual implica para las empresas y organizaciones que usan el conocimiento que quien no cambien e innove ira progresivamente quedándose atrás y desaparecerá su actividad como tal. En el ámbito de la tecnología se juega un rol importante en el ambiente de negocios, la comunicación global ahora es inmediata sin importar distancias, estas compañías confían en el software y computadores instalados para que su negocio sea más transparente, fácil de administrar y detectar nuevas oportunidades, también se considera como un modelo en la que una empresa produce, entrega y vende un producto o servicio para crear riqueza generando el crecimiento económico, fomento de la innovación y mejoría en el bienestar de los ciudadanos.

De acuerdo con Kast y Rosenzweig (1989), "la tecnología es la aplicación del conocimiento para el desempeño eficiente de ciertas tareas y actividades, convierte la conducta espontánea y no reflexionada en una conducta que es deliberada y racionalizada" (p.217). De otra forma, Bateman y Snell (2005) definen la tecnología como: "Los métodos, procesos, sistemas y habilidades que se utilizan para transformar recursos en productos. En una perspectiva amplia es posible considerarla como comercialización de la ciencia, la aplicación sistemática de conocimiento científico a un producto, proceso o servicio nuevo" (p.520). 
Por su parte, Koontz y Weihrich (1998) asumen que la tecnología se refiere a la suma total de conocimientos sobre la manera de hacer las cosas: diseñar, producir, distribuir, vender bienes y servicios. Según Escorsa y Valls (2005) menciona que las gestiones tecnológicas comprenden las actividades de gestión relacionadas con: identificación, obtención, investigación, desarrollo y adaptación de las nuevas tecnologías en la empresa.

Consideran que la gestión tecnológica se refiere al proceso gerencial de planear, organizar, dirigir y controlar la tecnología de una empresa con el fin de lograr los objetivos organizacionales.

- Planeación: función de la administración en la que se definen las metas, se fijan las estrategias para alcanzarlas, y se trazan planes para integrar y coordinar las actividades (Robbins y Coulter, 2005).

- Organización: según Robbins y Coulter (2005), hace referencia a la función de la administración que consiste en determinar qué tareas hay que hacer, quién las hace, cómo se agrupan, quién rinde cuentas a quién y dónde se toman las decisiones.

- Dirección: según Koontz y Weihrich (2004), dirigir es influir en las personas para que contribuyan a la organización y a las metas de grupo.

- Control: es la función de medir y corregir el desempeño individual y organizacional para garantizar que los hechos se apeguen a los planes (Koontz y Weihrich, 2004).

- La tecnología informática juega un papel importante en la toma de decisiones de la alta gerencia, se establece que la efectividad es el resultado de la valoración de tres aspectos fundamentales, que son: la eficiencia, la eficacia y la seguridad.

- La eficiencia: Implica la capacidad de obtener los mayores resultados con la mínima inversión (Robbins y Coulter, 2005).

- La eficacia: Implica completar las actividades para conseguir las metas de la organización; se define como "hacer las cosas correctas” (Robbins y Coulter, 2005).

Medellín (2010), manifiesta que las tecnologías establecen un marco de relaciones entre la empresa y los objetivos del negocio. La empresa cuenta con habilidades para la explotación de las oportunidades tecnológicas (capacidad de innovación), y esto le aporta valor a la empresa. Actualmente la innovación se considera un factor básico que no consiste únicamente en la 
incorporación de la tecnología, sino que requieren de actos emprendedores para su implementación y prosperidad para lo cual también se debe saber administrar la tecnología y sus recursos actuales para que no se queden atrás y prevalezcan, cuyo objetivo es incrementar la productividad y competitividad de las empresas a través del uso de herramientas tecnológicas basadas en estándares internacionales. La empresa, está soportada en los valores siguientes: innovación, integridad, calidad, trabajo en equipo, crecimiento, rentabilidad.

Ravina, Villena y Gutiérrez (2017), afirman que la economía globalizada e interconectada se establece como el verdadero motor de la innovación y transferencia de conocimiento científico, donde ambos factores contribuyen al impulso de la actividad productiva en la generación de estrategias innovadoras en bienes, servicios y procesos. Estas entidades destinan recursos monetarios fuertes, donde el desarrollo de la política de recursos humanos es con el compromiso colectivo de satisfacer el interés laboral ya que requiere de un entorno interno que aliente a la discusión y se realice un buen trabajo en equipo para satisfacer las necesidades humanas.

La productividad laboral en las empresas puede orientarse hacia un buen ambiente de trabajo mediante la adopción de determinadas acciones para gestionar el tiempo y los recursos o mejorar las capacidades de la empresa, tanto a nivel tecnológico como procesal. Una buena productividad de gestión empresarial favorable para lograr la sostenibilidad y la viabilidad futura de la empresa donde los términos de productividad y competitividad deben relacionase y depender mutuamente. La innovación mejora la competitividad de las empresas para hacer frente a un entorno cada vez más competitivo y más globalizado. Este fenómeno afecta a todas las empresas, independientemente de su tamaño o sector. En este sentido, Gargallo y Ramírez (2007) dan a conocer sobre las tecnologías que han pasado de ser un área de soporte y generadora de costos a ser una necesidad estratégica. Las empresas líderes son aquellas que innovan, tienen éxito y sus competidoras no les queda más remedio que imitarlas. Actualmente las pequeñas y medianas empresas se enfrentan a numerosos desafíos en una etapa de constantes innovaciones. Las Tecnologías de la Información y la Comunicación (TIC) permiten a las empresas mejorar su eficiencia.

Por ejemplo, la reducción de costes o la mejora de la calidad y del servicio ofrecido a los consumidores. Por tanto, la adopción de estas tecnologías resultará fundamental para la 
competitividad de las PYMES tanto por las oportunidades que ofrece como por las amenazas que se derivan para su supervivencia.

Díaz, (2008), manifiesta que las estrategias de gestión pueden apoyarse en soluciones tecnológicas, pensadas para responder en forma tal que permitan mejorar las oportunidades de supervivencia y el éxito en una época en que la fuerza corporativa se suele medir por el valor de los activos de información; conocimiento profundo del cliente; articulación de los procesos misionales de valor y de apoyo; oportunidad en las entregas, en la calidad y en los servicios post venta, que garantizan la solución de peticiones o reclamos.

Es necesario escoger las tecnologías más pertinentes para la empresa en las cuales invertir primero. El grado de relevancia de las tecnologías se puede evaluar según su contribución a la estrategia global de la empresa, los factores claves de éxito del mercado o las necesidades de los clientes. Cuando una nueva tecnología toma una importancia significativa son capaces de tomar la ventaja para mejorar su posici6n en la lucha competitiva. Díaz, Gonzales y Ruiz (2005) nos manifiestan como se realiza la implantación de un sistema ERP. El contar con tecnología que se adapte a los cambios constantes que se generan, es una ventaja competitiva que hace que las organizaciones sigan teniendo presencia en el mercado.

La decisión de invertir una cantidad considerable de capital en un proceso de implementación de un sistema de ERP refleja la disponibilidad de la administración para cambiar la manera tradicional de cómo opera la empresa. Las organizaciones que pueden adoptar y adaptarse a este sistema tienen una ventaja competitiva superior a las que no lo utilizan.

Molina (2005) comenta que tradicionalmente la economía ha girado en torno al sector de servicios; los grupos sociales favorecidos son los consumidores y tecnologías dominantes serán la informática, los nuevos materiales, la microelectrónica, la robótica y la biotecnología y el desarrollo económico tenderá a ser más sostenible y equilibrado. Las empresas de hoy se ven obligadas a tratar el tema de la tecnología como una variable central de la función productiva y a integrar la innovación tecnológica a la estrategia del desarrollo empresarial. La modernización empresarial es un reto tecnológico en el Mercado.

\section{6}

Pol. Con. (Edición núm. 30) Vol. 4, No 2, febrero 2019, pp. 407-424, ISSN: 2550 - 682X 
Díaz (2006), asegura que el uso efectivo de las herramientas tecnológicas facilita la identificación, crecimiento, flujo y creación de conocimiento de valor para la estrategia empresarial como un aspecto crítico, dado que han surgido múltiples técnicas computacionales cuyo análisis y comparación se dificulta, no solo por la relativa juventud de la gestión de conocimiento sino por el carácter multidisciplinario de la misma.

La gestión de conocimiento se dirige a los temas críticos de adaptación y supervivencia organizacional, así como la capacidad de ser competente frente a los cambios ambientales incrementales y discontinuos que caracterizan el ambiente empresarial.

Ramírez y Vega (2015) mencionan que el proceso de innovación no depende mayormente del avance tecnológico, sino del proceso generador de conocimiento y de las medidas ofensivas, permitiendo una clara diferenciación de los productos o servicios ofrecidos por la competencia. La innovación tanto en los productos, servicios y sus procesos de producción ha permitido ir alcanzado la madurez requerida para mantenerse en el mercado. Mediante la innovación, la organización optimiza sus procesos de producción, fortalece sus destrezas y adquiere valor agregado tanto para su marca o nombre, como para sus productos o servicios (Molina Penagos, 2005)

El foro económico representa una transición de un nuevo conjunto de sistemas que reúnen tecnologías digitales, biológicas y físicas en una moderna y poderosa combinación. Ya que permite la personalización total de los productos y la creación de nuevos modelos operativos.

Todos los nuevos desarrollos y tecnologías tienen una característica clave: aprovechan el poder omnipresente de la digitalización que brindan las tecnologías de la información y comunicación. Por su parte, Bernal y Saavedra (2012) nos manifiestan que es necesario destacar que las inversiones en equipo de producción, capital de trabajo, innovación, investigación, desarrollo y capacitación sí utilizaron apalancamiento financiero mediante créditos bancarios, lo que perciben como generador de valor.

\section{Metodología}

Para cumplir con el propósito central formulado en este artículo, se propuso una metodología que 
combina elementos de la investigación documental y descriptiva de carácter retrospectivo al fundamentar las teorías relacionadas a las tecnologías avanzadas de gestión empresarial y modelado en planteamientos evaluados y examinados por estudiosos del tema, así como la experiencia de investigadores del área de la tecnologías e información desde la web, todos dentro de un tiempo considerado lo suficientemente amplio para obtener datos registrados de documentos escritos y conclusiones históricamente significativas que permitieron presentar conclusiones válidas para el estudio.

\section{Conclusiones}

A continuación, se presenta la interpretación de las teorías abordadas y referidas a las tecnologías avanzadas de gestión empresarial y modelado, las cuales condujeron a generar conclusiones concretas y precisas de las mismas, entre estas:

- La tecnología ha sido un proceso acumulativo clave en la experiencia humana, ha progresado por el método empírico del tanteo y ha estado a la vanguardia en muchos campos. Es común escuchar que algunos países no se pueden adaptar a las nuevas tecnologías ya que corren el riesgo fatal de quedarse al margen en términos de desarrollo y bienestar.

- La ciencia y la tecnología son productos humanos, como tales incorporan también los valores y los intereses propios, en cuyo desarrollo se integran de un modo muy sutil a lo que debe ser. Continuamente interaccionan las controversias sociales y sus implicaciones en la formación de los profesionales en la gestión empresarial.

- También, es necesario estudiar los acuerdos comerciales entre países ya que modifican las condiciones del mercado mediante la supresión o disminución de aranceles o tasas; las políticas fiscales del país que favorezcan la inversión en nuevas tecnologías. Dado que el valor de una empresa apalancada, es superior al de una empresa no movible, por el beneficio fiscal que recibe por medio de la deducción de los intereses de la deuda. 
- La integración de la tecnología en las labores empresariales hará que se den mejores resultados y se genere una mejor aceptación en los diferentes mercados. Se debe tener en cuenta que la tecnología progresa rápidamente, razón por la cual, las personas se deben actualizar constantemente.

- El gerente es el encargado de hacer que las empresas funcionen correctamente y debe tener un sin número de características para poder lograr un buen trabajo y generar eficiencia y eficacia, también desempeñan una labor importante dentro de las empresas ya que son los que analizan las situaciones por las que pasan las mismas y a la vez, toman decisiones. Siempre deben enfocarse en generar innovación y competitividad, para esto, se debe optar por integrar tecnología dentro de la empresa, logrando una ventaja competitiva.

- La introducción e innovación de nuevas tecnologías frente al entorno económico, avance tecnológico y los cambios en políticas de comercio internacional obligan a las empresas globales a alcanzar y mantener una ventaja competitiva que cada vez es más difícil mantenerla. Los componentes relacionados a los nuevos negocios internacionales tienen que ir de la mano con avances y hacer frente a las tendencias globalizadoras para conseguir ventajas competitivas.

- El uso de la tecnología en las empresas acerca más a las personas y sus culturas y que al invertir en tecnología de información y comunicaciones tienen más probabilidad de éxito en un futuro.

- La innovación tecnológica se ha introducido en las empresas no solo como una herramienta para la solución de problemas con empleados, proveedores o clientes, sino que han fomentado un ambiente de colaboración, al compartir soluciones, mejoramiento continuo en tiempo real, así como mayor y mejor atención a clientes, de la misma manera ofrecer bienes o servicios de mejor calidad, esto significa e implica una atomización de los procesos de negocio y un aumento de la competitividad. Los usos de estas nuevas tecnologías deben verse como una potente herramienta que ayuda a implementar nuevas formas de mejorar frente a las competencias y sobresaliendo cada vez más hasta conseguir resultados favorables para la empresa. 
- La gestión tecnológica en la empresa se refiere al proceso de planeación, organización, dirección y control. Los gerentes deben promover las acciones hacia: la integración de la gestión tecnológica con la estrategia global de la empresa, la vigilancia de los avances tecnológicos, la selección de la tecnología para las diferentes actividades, el resguardo del Know-How, el impulso a la innovación y la vinculación con las universidades.

- La tecnología puede generar la optimización de los recursos y, por tanto, mayor beneficio para la empresa. La TIC sirve de apoyo para lograr la competitividad de la organización, y realizar una correcta toma de decisiones en el contexto de la gestión tecnológica. El modelo para evaluar la efectividad de la TI permite analizar el costo de inversión en innovación tecnológica y los beneficios que esta puede brindar para la organización y permitirá dar una visión con objetividad sobre las implicaciones que tiene la implantación de la TI en procesos administrativos.

- La implantación de un sistema ERP es necesario en una organización ya que ayuda a la organización con procesos positivos y eficaces, con la habilidad de la tecnología en la adaptación de los cambios constantes, para que las organizaciones sigan teniendo presciencia en el mercado.

- Las pequeñas y medianas empresas están en desafíos de constantes innovaciones en todos los ámbitos. Las TICS permiten a las empresas mejorar su cargo, de modo que la adopción de las tecnologías resulta importante para la competitividad de las pequeñas y medianas empresas (PYMES).

- La revisión de los factores que influyen en la adopción de las TIC en las organizaciones, sintetizando factores de entorno, organizacionales, tecnológicos y las características propias del gerente propietario, ha permitido destacar la importancia de su estudio conjunto de modo que al conocer los elementos claves que favorecen el acceso de las pequeñas y medianas empresas a las TIC no es suficiente. Pero lo más necesario es que las PYMES desarrollen y fortalezcan sus capacidades para llevar a cabo un correcto ajuste entre estas tecnologías y el resto de elementos de la organización. 
- Las guías de los sistemas de información que ofrecen las TIC, no son excluyentes entre sí, pero su tratamiento secuencial ha permitido identificar de manera gradual retos y oportunidades en la industria de la información y gestión del conocimiento.

- La búsqueda de los aspectos comunes de las dimensiones del conocimiento analizadas estuvo motivada en acompañar a las empresas en su desarrollo y evolución, incorporando prácticas modernas de gestión empresarial.

- Las organizaciones deben su razón de ser al hecho de poder desarrollar su existencia dentro del entorno, a través del cual sus clientes pueden acceder a los servicios y productos, pero por componentes globalizadores, así como también la competencia han impuesto una mejora continua de los productos, servicios que comercializan y mantienen su vigencia.

- La organización mediante la innovación permite mejorar la producción y adquirir un valor agregado para la marca y el nombre del producto o servicio que permiten adquirir una madurez para mantener dentro del mercado.

- Los SIG ha sido una herramienta desarrollada para la potencialización de las telecomunicaciones previstas por la Web, así como también mejorar la flexibilidad de las operaciones para disponer de una adecuada base de datos y a su vez generan un bajo costo. Asimismo, se han ido desarrollando desde la perspectiva hacia lo interno, a establecerse como un eslabón de interrelación de la organización con un entorno cada vez más demandante de valor agregado. Se han potenciado por el desarrollo vertiginoso del mundo de las telecomunicaciones provistas por la Web y mediante el cual se han consolidado todas estas relaciones a un bajo coste.

\section{Referencias Bibliográficas}

Bateman, T y Snell, S. (2005). Administración. Un nuevo panorama competitivo. México D.F... México. $6^{\mathrm{a}}$ ed. McGraw-Hill Interamericana

Barragán J y Vela J. (2015). Impacto de las TICS en la Gestión de Negocios Internacionales. Recuperado de http://www.spentamexico.org/v10-n3/A3.10(3)18-33.pdf 
Bernal, D y Saavedra, M. (2012). Inductores de valor empresarial y decisiones de inversión. Un análisis sectorial. Actualidad Contable FACES Año 15 N² 25, Julio - Diciembre 2012. Mérida. Venezuela (26-40)

Díaz, A; Gonzalez, J; y Ruiz, M. (2005) Impacto de un sistema ERP en una organización) Recuperado de: http://revistasinvestigacion.unmsm.edu.pe/index.php/sistem/article/download/3475/2862.

Díaz, L (2006). Gestión del conocimiento y tecnología de información y comunicaciones. Escuela de Administración de Negocios, núm. 58, septiembre-diciembre, 2006, pp. 41-59 Universidad EAN Bogotá, Colombia.

Escorsa, P y Valls, J (2005). Tecnología e innovación de la empresa. España. Ediciones UPC.

Gargallo A, y Ramirez M, (2007) La adopcion de las tecnologias de la información en las pequeñas y medianas empresas) Recuperado de: http://www.redalyc.org/html/4777/477748626003/.

Kast, F.; Rosenzweig, J. (1989). Administración en las Organizaciones. Enfoque de Sistemas y de Contingencias. México: McGraw-Hill/Interamericana de México, S.A. de C.V.

Koontz, H y Weihrich, H. (2004). Administración. Decima Octava ed. México. Mc Graw Hill.

Koontz, H., y Weihrich, H. (1998). Administración. (Octava Ed.). (Edit, Trad.) Mc Graw Hill.

Marcovitch, S. (2001). Sobre la necesidad de control consciente y entendimiento conceptual. Ciencias del comportamiento y del cerebro. 2001; 24: 48-49.

Martínes, M. (2006) La gestión de la innovación tecnológica en pequeñas y medianas empresas (PYMES). Recuperado de https://www.redalyc.org/pdf/141/14111976008.pdf

Medellín, E.(2010). Gestión tecnológica en empresas innovadoras Mexicanas. Universidade de São Paulo, Brasil: RAI - Revista de Administração e Inovação. Recuperado de: https://www.redalyc.org/html/206/20605804/ 
BIBLIOGRAPHY Molina Penagos, R. (2005). ¿Por qué gerenciar el factor tecnológico en la empresa?

EAN.

Recuperado de:/C:/Users/DREDDCOM0991271159/Documents/tecnologia1.pdf

Núñez, E (2011). Gestión tecnológica en la empresa: definición de sus objetivos fundamentales Revista de Ciencias Sociales (Ve), vol. XVII, núm. 1, enero-marzo, 2011, pp. 156-166 Universidad del Zulia Maracaibo, Venezuela

Perel, V (2015). IMPACTO DE LA TECNOLOGÍA EN LAS ORGANIZACIONES Tema: "Los retos del futuro: Tecnología y personas" XII Congreso Internacional de Administración C.P.C.E.A.B.A., 11 al 13 de noviembre de 2015

Perozo, E y Nava, A (2005). Determinantes de competitividad empresarial. Caso de pequeñas y medianas empresas. \}Recuperado

de: http://congreso.investiga.fca.unam.mx/docs/xvii/docs/C09.pdf

Ramírez, J y Vega, O. (2 de julio-diciembre de 2015). SISTEMAS DE INFORMACIÓN GERENCIAL E INNOVACION PARA EL DESARROLLO DE LAS ORGANIZACIONES. Universidad Privada Dr. Rafael Belloso Chacín, Zulia, Venezuela: Télématique. Obtenido de http://www.redalyc.org/articulo.oa?id=78440280006

Ravina R, Villena F y Gutiérrez, R (2017) Una Aproximación Teórica para el mejor resultado de Innovación en las Empresa Documento: HYPERLINK "http://doi.org/10.17163/ret.n14.2017.06" It "_blank" http://dx.doi.org/10.17163/ret.n14.2017.06 .

Robbins y Coulter, M. (2005). Administración. 8va Edición. México. Pearson

Díaz, F. (2008). Gestión de procesos de negocio BPM (Business Process Management), TIC y crecimiento empresarial ¿Qué es BPM y cómo se articula con el crecimiento empresarial? Universidad Católica de Colombia 
Zamora, J. (2013). Las nuevas tecnologías y su impacto en la competitividad empresarial. Recuperado de: https://www.iese.edu/es/noticias/las-nuevas-tecnologias-y-su-impacto-en-lacompetitividad-empresarial/

Zapata, A; Arango, M.D. y Adarme, J (2010) Herramientas tecnológicas al servicio de la gestión Empresarial Arquitectura Empresarial - Una Visión General. Revista Ingeniería Universidad de Medellín. Número 16. 